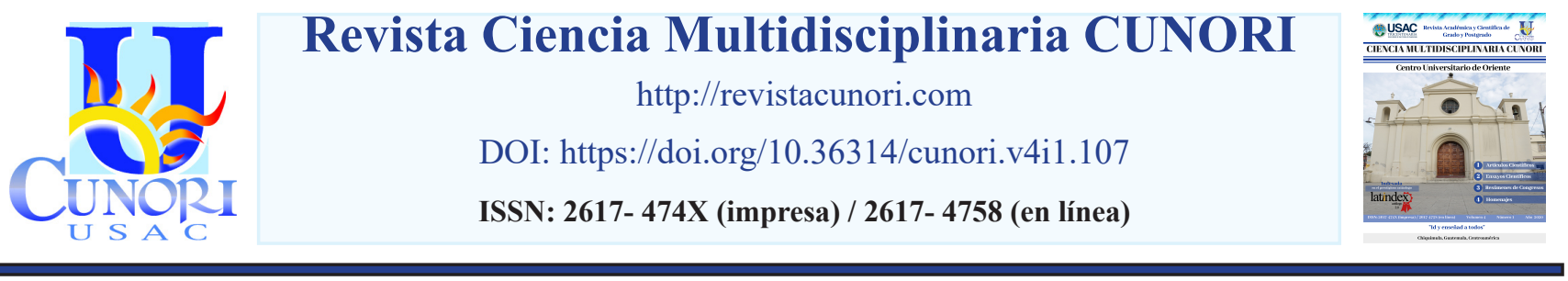

Como citar el artículo

Recinos, Y. (2020). Los objetivos de desarrollo sostenible, una estrategia para evitar la desnutrición materna. Revista Ciencia Multidisciplinaria CUNORI, 4(1), 29-42. DOI: https://doi.org/10.36314/cunori.v4i1.107

\title{
Los objetivos de desarrollo sostenible, una estrategia para evitar la desnutrición materna
}

\author{
The sustainable development goals, a strategy to avoid maternal undernutrition \\ Yessenia Eunice Recinos Méndez \\ Centro Universitario de Suroriente (CUNSURORI, Universidad de San Carlos de Guatemala \\ Recibido: 08 de marzo de 2020 / Revisión: 25 de abril de 2020 / Aceptado: 10 de mayo de 2020 \\ Disponible en internet el 29 de mayo de 2020 \\ *Autor para correspondencia. \\ Correo electrónico: yessenia_recinos@yahoo.es
}

Resumen

T a desnutrición materna es una problemática multifactorial que a afecta directamente a la población en general al dar como _resultado restricciones del crecimiento intrauterino, recién nacidos de bajo peso y aumentando la mortalidad materno-infantil. El objetivo del presente artículo es identificar cuáles de los objetivos de desarrollo sostenible pueden ayudar a disminuir la desnutrición en las embarazadas. Metodología: Para realizar la revisión del tema se identificaron artículos publicados en español tanto en Revistas como en medios electrónicos. Resultados: los objetivos: Fin de la pobreza, hambre cero, salud y bienestar, educación de calidad, igualdad de género, Trabajo decente, crecimiento económico y Reducción de las desigualdades tienen una influencia directa en disminuir la desnutrición en general y de las embarazadas. Conclusión: Existe un alto riesgo que al igual que no se cumplieron los objetivos del milenio no se logre alcanzar los objetivos del desarrollo sostenible.

Palabras clave: objetivos de desarrollo sostenible, desnutrición en embarazadas, restricción del crecimiento intrauterino, bajo peso al nacer

\begin{abstract}
$\mathrm{M}$ aternal malnutrition is a multifactorial problem that directly affects the population in general, resulting in intrauterine growth restrictions, low birth weight babies and increasing maternal and infant mortality. The objective of this article is to identify which of the sustainable development objectives can help reduce malnutrition in pregnant women. Methodology: To review the topic, articles published in Spanish were identified in both Journals and electronic media. Results: the objectives: End of poverty, zero hunger, health and well-being, quality education, gender equality, decent work, economic growth and reduction of inequalities have a direct influence on reducing malnutrition in general and pregnant women. Conclusion: There is a high risk that, just as the Millennium Development Goals were not met, the objectives of sustainable development will not be achieved.
\end{abstract}

Keywords: sustainable development goals, malnutrition in pregnant women, intrauterine growth restriction, low birth weight 


\section{Introducción}

La desnutrición materna es un tema relevante no solo a nivel de medicina sino a nivel social, la desnutrición en embarazadas puede llegar a tener consecuencias devastadoras ya que esta puede influir en el desarrollo de las futuras generaciones.

El peso y la talla de las madres son factores predisponentes para tener hijos con adecuado peso o bajo peso al nacer (Delgado, Roca, Suárez, Rodríguez., \& Ruiz, 2012), el estado nutricional de las embarazadas no es el único que predispone a ello, pero si uno de los principales, ya que las mujeres de baja estatura y con desnutrición durante el embarazo son la principal causa del retardo del crecimiento intrauterino, y este es a su vez una causa directa de la muerte neonatal entre otros. (Pimiento \& Beltrán, 2015).

Existen múltiples estrategias que buscan erradicar la desnutrición materna y neonatal, entre estas se encuentran los Objetivos de Desarrollo Sostenible (ODS), los cuales constituyen una segunda etapa estratégica de desarrollo planteada por las Naciones Unidas (NU) con el propósito de crear un conjunto de objetivos mundiales relacionados con los desafíos políticos, económicos y ambientales, estos vienen a sustituir a la primera etapa que fueron los Objetivos de Desarrollo del Milenio creados en el año 2000 con la finalidad de hacer frente a la pobreza extrema y al hambre, prevenir enfermedades mortales y ampliar la enseñanza primaria a todos los niños entre otras, metas que no se lograron en su totalidad para el año 2015 (Programa de las Naciones Unidas para el Desarrollo PNUD, 2015). Siendo Guatemala uno de los países que quedo muy lejos de conseguirlos y quedando comprometida con la población en general y a nivel mundial para poder alcanzarlos.

Los ODS se gestaron en la Conferencia de las Naciones Unidas sobre el Desarrollo Sostenible, celebrada en Río de Janeiro en 2012, constituyendo un total de 17 objetivos, 169 metas las cuales se monitorean a través de 241 indicadores que buscan resolver los problemas multidimensionales de la pobreza, abordando temas de carácter universal y estratégicos para el desarrollo, estos fueron aprobados por 193 países incluidos Guatemala y se busca alcanzar para el año 2030. (SEGEPLAN, 2017)

Los objetivos de desarrollo sostenible tienen como fin primordial liberar a la humanidad de la pobreza, asegurar un planeta sano para las siguientes generaciones, y construir sociedades incluyentes y pacificas para garantizar vidas dignas, teniendo como visión un mundo más justo, próspero y sostenible (Organización de las Naciones Unidas para la alimentación y la agricultura FAO, 2015). Estos tienen estrategias importantes como: promover la igualdad de género, en los cuales se les da valor a los derechos de las mujeres para que tengan acceso a la educación, a la alimentación y a poder decidir sobre ellas mismas, lo que influye directamente en la sociedad, en la familia y en lo individual. La educación en las mujeres asegura familias más desarrolladas, al lograr erradicar el hambre en todas las personas se logrará erradicar la desnutrición proteico-calórica y promover poblaciones sanas. (PNUD, 2018a.)

\section{Materiales y métodos}

Se ha realizado una revisión sistemática de documentos relacionados con los Objetivos del Milenio, Objetivos de Desarrollo Sostenible, Desnutrición Materna y neonatal, la búsqueda se hizo tanto en fuentes 
físicas como en medios electrónicos, se han localizado un total de 102 bibliografías, de las cuales se han incluido 47 que fueron relevantes para esta investigación.

\section{Desarrollo y discusión}

\section{Desnutrición}

La desnutrición sigue siendo una amenaza para la supervivencia, la salud, el crecimiento y el desarrollo de los niños, al igual que para el progreso de los países. (Wisbaum, 2011). Según la Organización Panamericana de la Salud (OPS, 2009) esta es causa directa de alrededor de 300 mil muertes en el año e indirectamente responsable de la mitad de las muertes en menores de 5 años.

La desnutrición se puede presentar en cualquier etapa de la vida, desde el retraso de crecimiento intrauterino hasta la desnutrición crónica del adulto. El retraso en el crecimiento intrauterino, la desnutrición crónica y la desnutrición aguda severa a nivel mundial contribuyen con 91 millones de años de vida saludable perdidos, 2.2 millones de muertes por año y es responsable del 7\% de la carga total de enfermedad (OPS, 2009), siendo la desnutrición un problema multisectorial y multicausal (Comisión Nacional para la Reducción de la Desnutrición Crónica, 2016), abarcando la alimentación, salud y prácticas de atención.

\section{Desnutrición en Guatemala}

El Estado de Guatemala, garantiza y protege la vida humana desde su concepción, así como la integridad y la seguridad de la persona, (Constitución Política de la República, 1993). El derecho a la alimentación encuentra su desarrollo normativo principalmente a través de diferentes leyes, entre ellas: la Ley del Sistema Nacional de Seguridad Alimentaria y Nutricional (2005), la cual indica que el acceso físico, económico y social a la alimentación con pertinencia cultural y en cantidad y calidad adecuada es un derecho de toda persona, dándosele preferencia a lo nacional.

La Ley de Protección Integral de la Niñez y la Adolescencia (2003), la Ley de Protección para las personas de la tercera edad (1996) y el Código de Salud, (1997). Pero a pesar de ello la desnutrición se encuentra presente. Según Maggie Fisher (2015) Guatemala, a nivel mundial, ocupa el sexto lugar de desnutrición crónica y la mayor prevalencia en las Américas, dicha malnutrición afecta la salud de mujeres y niños, comprometiendo el capital humano y los esfuerzos de desarrollo en general.

Según la UNICEF (2010): Cuatro de cada diez niños y niñas guatemaltecos menores de 5 años (43.4\%) presentaba desnutrición crónica (talla en relación a la edad), afectando a 8 de cada 10 niños y niñas indígenas con consecuencias que pueden llagar desde la falta de retención escolar, individuos menos productivos, vulnerabilidad para las enfermedades, hasta perdida del coeficiente intelectual.

En datos aportados por Gobierno de Guatemala en el cuarto censo nacional de talla (2015), valores promedio nacional de la prevalencia de desnutrición crónica en escolares eran del $37.6 \%$ en 2015 la cual aún es muy alta según los parámetros usados por la Organización mundial de la salud (OMS) variando 
en cada departamento, pero también hubo datos alentadores, los cuales al comparar los valores de las prevalencias de desnutrición crónica o retardo en talla para edad, disminuyeron en 1.3 puntos porcentuales de 1986 a 2001, hasta 8.0 puntos porcentuales de 2008 a 2015.

\section{Desnutrición en embarazadas}

El estado nutricional de la embarazada está determinado en función de tres parámetros: talla, peso y edad gestacional, midiéndose a través del Índice de Masa Corporal (IMC) (Sánchez, Trelles., Castanedo \& Lugones, 2003). Según la ENSMI (2017) Los criterios que se utilizan para interpretar los resultados del IMC son: valor menor de 18.5 se clasifica como bajo peso; valor entre 18.5 y 24.9 se clasifica como normal; valor de 25 a 29.9 se clasifica como sobrepeso y valor de 30 o más se considera obesidad.

En Guatemala de las mujeres en edades de 15 a 49 años un $45 \%$ se encuentran con un IMC normal, el grupo con mayor normalidad es el de 15 a 19 años teniendo un $71 \%$ y este disminuye conforme aumenta la edad (ENSMI, 2017)

El conocimiento del estado nutricional de las mujeres es importante ya que este influye directamente en su salud, a partir de este se puede llegar a padecer enfermedades crónicas no transmisibles que alteran la productividad. Según el Instituto de Nutrición de Centro América y Panamá (INCAP, 2017), existe una relación estrecha entre el estado nutricional y los riesgos que se presentan en el embarazo, el parto y el peso que lleguen a tener sus hijos al nacer.

La estatura baja en los padres, y la desnutrición durante la gestación son la principal causa del retardo de crecimiento intrauterino (Delgado et al., 2012), esta es multifactorial, y representa altas tasas de morbilidad materna y perinatal (Pimiento et al., 2015). La contribución de la desnutrición materno-infantil a la mortalidad infantil es de más de un tercio, (Rivera, Martorell, González, Lutter, Cossío, Flores-Ayala \& Delgado, 2011).

Los recién nacidos con bajo peso al nacer tienen hasta 5 veces mayor probabilidad de morir entre el primer mes y el primer año de vida comparado con los que nacen con peso normal, en Guatemala el bajo peso al nacer es del 41\%, siendo los factores preconcepcionales el bajo nivel socioeconómico-educacional de la mujer entre otros. (Rebollar, Ávila \& Gutiérrez, 2010) Es por ello que se debe de insistir en que se trabaje arduamente en estos problemas.

\section{De los objetivos del milenio a los objetivos de desarrollo sostenible}

Para abordar los objetivos de desarrollo Sostenible se debe de partir de los objetivos de desarrollo del milenio que los antecede. Los objetivos del milenio se firmaron en Nueva York en septiembre del 2000 donde 189 líderes mundiales se comprometen a liberar de las condiciones de extrema pobreza a la población en general antes del 2015 a hombres mujeres y niños, siendo esta la consigna principal. (Secretaría de Planificación y Programación de la Presidencia SEGEPLAN, 2016). 
De los 8 objetivos de desarrollo del milenio que se plantearon para ser cumplidos del año 2000 al 2015 Guatemala informó lo siguiente: En reducción a la mitad el número de personas viviendo en pobreza extrema, las personas que no tienen acceso a agua potable y reducir el hambre, no fue cumplida ya que únicamente $41 \%$ de la población no vive en pobreza.

En el objetivo de lograr la enseñanza primaria universal: la meta era llegar a un 100\% logrando únicamente en 2014 un 82\%, aunque hubo avances en este tema no se logró llegar a lo pretendido. En relación a Promover la igualdad entre los géneros y la autonomía de la mujer: la meta era 95\% y únicamente se alcanzó a un 50\%. Reducir la mortalidad infantil en dos tercios; fue satisfactorio haberla logrado. Con respecto a mejorar la salud materna y reducir la mortalidad materna en tres cuartos; aunque se redujo en un $50 \%$ en comparación con el año base, el indicador no se cumplió. Mientras que los objetivos de: Combatir el VIH/SIDA, el paludismo y otras enfermedades; Fomentar una asociación mundial para el desarrollo, no fueron alcanzados, garantizar la sostenibilidad del medio ambiente este objetivo evolucionó negativamente (SEGEPLAN, 2015)

En septiembre de 2015 se realizó la reunión de alto Nivel de las Naciones unidas con la Declaración de Transformar Nuestro Mundo: La agenda 2030 para el desarrollo sostenible donde Guatemala oficialmente adoptó los Objetivos de Desarrollo Sostenible (SEGEPLAN 2017)

Guatemala asumió en su momento los objetivos del milenio como Estrategia de desarrollo, mientras que los Objetivos de Desarrollo Sostenible el gobierno actual los alinea al Plan Nacional de Desarrollo K'atun: Nuestra Guatemala 2032 que es su estrategia de desarrollo de largo plazo. (SEGEPLAN, 2016) con el fin de incluirlos dentro de la agenda de trabajo. De los17 ODS se puede identificar que: el fin de la pobreza, hambre cero, salud y bienestar, educación de calidad, igualdad de género, trabajo decente, crecimiento económico y reducción de las desigualdades, tienen influencia directa en disminuir la desnutrición en general y de las embarazadas.

Según la Organización de las Naciones Unidas (ONU, sf), lograr un mundo más igualitario para hombres y mujeres es una de las mejores oportunidades para afrontar desafíos urgentes, reducir la pobreza infantil, lograr el acceso a educación para los todos los niños, entre otros, es un programa ambicioso en el cual se deben de comprometer todos los países.

\section{Fin de la pobreza}

Poner fin a la pobreza en todas sus formas en todo el mundo es el objetivo número uno de los 17 objetivos planteados en los ODS, lograr que todas las personas tengan recursos adecuados para suplir sus necesidades básicas es vital para el desarrollo individual y de sociedad. Según las Naciones Unidas (NU, 2016) desde 1990 los índices de pobreza extrema se han reducido a la mitad, pero aun así 1 de cada 5 personas de las regiones en desarrollo sigue viviendo con menos de 1,25 dólares al día, lo que expone a una gran cantidad de personas de caer en pobreza extrema impidiendo que se tenga una nutrición adecuada. 
Según el Banco de Guatemala para el año 2016 el país tenía 16,548,168 de habitantes, de los cuales $59.28 \%$ se encontraba en pobreza ( $23.33 \%$ en pobreza extrema, $35.93 \%$ en pobreza no extrema) y un $40.72 \%$ en no pobreza. Lo cual hace que la población no pueda adquirir los recursos necesarios para tener una vida digna exponiéndola a mayores riesgos y haciéndola más vulnerable. Mientras que en el informe realizado por el este mismo banco en 2015 indica que en el año 2011 la pobreza total era de $53.7 \%$ (el 18\% se encontraba en pobreza extrema) y el $47 \%$ en no pobreza lo cual indica que a pesar de los esfuerzos realizados la pobreza en Guatemala sigue empeorando, entonces es momento de definir que se está haciendo mal.

\section{Hambre cero}

Poner fin al hambre y asegurar el acceso de todas las personas a una alimentación sana, nutritiva y suficiente, sobre todo a los que se encuentran en pobreza y en situaciones vulnerables así como poner fin a todas la formas de malnutrición, buscando disminuir el retraso de crecimiento y la emaciación de los niños menores de 5 años abordando las necesidades de nutrición de las adolescentes, mujeres embarazadas y lactantes son metas priorizadas para este objetivo en Guatemala. (SEGEPLAN, 2017)

La alimentación adecuada es base para garantizar el bienestar humano y el potencial de cada individuo, por lo que es derecho de todos los hombres, mujeres y niños tener una nutrición adecuada, especialmente en la etapa denominada la ventana de los 1,000 días, la cual consiste en la etapa desde el embarazo hasta los 2 años de vida (Scalin up Nutrition, 2015) la alimentación debe de ser suficiente y nutritiva durante todo el año, (PNUD, 2018b) para continuar con la disminución de la mortalidad infantil prevenible.

Las metas convenidas internacionalmente sobre el retraso del crecimiento y la emaciación de los niños menores de 5 años, y abordar las necesidades de nutrición de las adolescentes, las mujeres embarazadas y lactantes buscan erradicar las consecuencias en la población a futuro, ya que la desnutrición en adolescentes hace que lleguen desnutridas al embarazo teniendo como consecuencia niños desnutridos. (Peraza, 2001), por lo que se debe de garantizar una vida sana y promover el bienestar en todas las edades.

A diferencia de otros países en los cuales la edad fértil es considerada de 14 a 44 años, en Guatemala esta etapa es de los 10 a los 54 años (Ministerio de Salud Pública y Asistencia Social MPSAS, 2010.), el embarazo es fisiológico pero expone a la mujer, al feto y al recién nacido a la probabilidad de enfermar o morir, siendo esta probabilidad mayor si se presenta desnutrición en la madre o en el recién nacido, (Donoso, Carvajal, Vera, \& Poblete, 2014) por lo cual la adecuada alimentación en niñas y adolescente es importante, para que al llegar a la etapa de la maternidad se encuentre en adecuadas condiciones nutricionales.

El estado nutricional de la mujer embarazada tanto antes y durante el embarazo es definitivamente un factor fundamental para la salud de ella y de su hijo, (Mendoza, Pérez, \& Bernal, 2010). Según Grados, Valderrama, Cabrera, y Díaz, (2003), el estado nutricional antes del embarazo y la ganancia de peso que logre durante este son los dos factores relacionados con el peso al nacer y este con la morbimortalidad perinatal, el crecimiento antropométrico y el desarrollo mental del recién nacido. 
En Guatemala el sistema nacional de seguridad alimentaria y nutricional ha sido fortalecido a través de varios gobiernos, aunque ha habido mejorías en algunos indicadores, las metas establecidas en convenios internacionales no se han alcanzado. (Vivero, 2014)

Tabla No. 1.

Avances de los sistema nacional de seguridad alimentaria y nutricional

\begin{tabular}{|c|c|c|c|}
\hline Presidente & Mandato & Instituciones & Políticas \\
\hline Alfonso Portillo & $2000-2003$ & $\begin{array}{l}\text { VISAN (MAGA), } \\
\text { CONSAN }\end{array}$ & Política SAN 2000 \\
\hline Oscar Berger & 2004-2007 & $\begin{array}{l}\text { SESAN, CONASAN, } \\
\text { Ley SAN, INCOPAS }\end{array}$ & $\begin{array}{l}\text { PRDC, Política SAN } \\
2005\end{array}$ \\
\hline Álvaro Colon & $2008-2011$ & Mesas Sectoriales SAN & $\begin{array}{l}\text { Bonos Solidarios } \\
\text { PESAN 2012-2016 }\end{array}$ \\
\hline Otto Pérez & $2012-2015$ & $\begin{array}{l}\text { Acuerdo Ventana } 1000 \\
\text { días, VAPS (MSPAS) }\end{array}$ & $\begin{array}{l}\text { Pacto Hambre Cero } \\
\text { Gestión x Resultados }\end{array}$ \\
\hline Jimmy Morales & 2016-2019 & $\begin{array}{l}\text { Estrategia Nacional para } \\
\text { la Prevención de la } \\
\text { Desnutrición Crónica } \\
2016-2020\end{array}$ & $\begin{array}{l}\text { Plan Nacional de } \\
\text { Desarrollo K'atun }\end{array}$ \\
\hline
\end{tabular}

Fuente: Vivero, J. (2014), SEGEPLAN, (2016).

\section{Salud y bienestar para todos}

El abordaje de la salud en general es el tercer objetivo de los ODS, el cual busca garantizar una vida sana y promover el bienestar de todos a todas las edades, especialmente las prioridades sanitarias prevenibles, y la salud reproductiva, materna e infantil.

Según SEGEPLAN (2017) entre las metas planteadas para este objetivo se encuentran: reducir la tasa mundial de mortalidad materna a menos de 70 por cada 100.000 nacidos vivos, debiendo recordar que Guatemala en 2014 logró disminuir la mortalidad materna en un 50\% pero esto fue únicamente 115 por cada 100,000 nacidos vivos por lo cual se sigue trabajando para reducirla, otra meta es poner fin a las muertes evitables de recién nacidos y de niños menores de 5 años, garantizar el acceso universal a servicios de salud sexual y reproductiva, incluidos la planificación de la familia, la información, la educación, y la integración de la salud reproductiva en las estrategias y los programas nacionales. 
A pesar de los esfuerzos que se están realizando, según las Naciones Unidas (2017) la tasa de mortalidad materna mundial se redujo en un 37\%, y la de los menores de 5 años cayó en un 44\%, entre los años 2000 y 2015, aunque la mayoría de muertes fueron prevenibles, en estos objetivos se está logrando hacer avances positivos.

El acceso a la salud debe de mejorarse, dado que muchas mujeres inician sus embarazos con bajo peso y no tienen acceso a un adecuado control prenatal por lo que no es posible que logren la ganancia adecuada de peso cada mes y poder evitar las consecuencias secundarias a ello.

\section{Educación de calidad e igualdad de género}

El nivel de escolaridad de mujeres y hombres, especialmente el acceso de la mujer a la educación, es uno de los determinantes principales de desarrollo de un país, logrando tener cambios en las practicas relacionados en la salud reproductiva e infantil (ENSMI 2017).

Logrando garantizar una educación inclusiva, equitativa de calidad y promover oportunidades de aprendizaje durante toda la vida para todos, permite que se pueda adquirir conocimientos necesarios para el desarrollo individual y familiar, es necesario que en todas partes del mundo, las mujeres y las niñas tengan derechos y oportunidades iguales y puedan llevar una vida libre de violencia y discriminación (ONU Mujeres, 2017), se ha determinado que el nivel de educación de las mujeres influye en el bajo peso al nacer, ya que la educación y la salud van de la mano, las niñas que logran un mayor nivel de educación, se embarazan más tardíamente y tienen menor número de hijos, lo que las hace madres con un nivel de madurez mayor. (Chan, 2010).

En Guatemala las brechas que existen entre la educación de hombres y mujeres está disminuyendo, igualmente que el analfabetismo, observándose un aumento en las personas que terminan la educación secundaria. Las brechas de genero se están cerrando cada vez más, en el reporte realizado por la VI ENSMI (2017) la brecha es de 2.2 por ciento en la población d 40 a 44 años con educación superior y para el grupo de 25 a 29 años esta brecha es del $0.9 \%$ es decir que las mujeres cada vez más van alcanzando mayor nivel de educación que en años anteriores.

Los derechos sexuales y reproductivos son fundamentales, ya que al privar a las mujeres de ellos se producen otras formas de discriminación, privándolas de educación y de trabajo decente (Morlachetti, 2007).

Según la ley de planificación familiar de Guatemala (2005), al garantizar el acceso universal a salud reproductiva y sexual les da a las madres el poder de decidir cuándo y cuantos hijos tener y así dedicarles mayor tiempo a cada uno de ellos para un crecimiento adecuado, es decir ayuda a cuidar la salud y vida de la madre y sus hijos, lo cual se está logrando a través de esta ley, logrando empoderar a las mujeres de esto y logrando que el uso de métodos anticonceptivos haya mejorado ya que según las estadísticas nacionales en 1987 el uso de métodos anticonceptivos era de un 23\% y para 2015 llego a ser de un 60\% (ENSMI, 2017). 
La evidencia sugiere que en los países de fecundidad alta al no tener la información y acceso a los servicios de salud reproductiva y debido a las barreras sociales y culturales que existen, limitan el ejercicio pleno de la vida reproductiva de dichas mujeres. (Flores, \& Soto, 2008).

De acá la importancia de la educación inclusiva, ya que la educación es uno de los motores más poderosos y probados para garantizar el desarrollo sostenible, por lo que se persigue que para el año 2030 todas los niños y niñas alcancen la educación secundaria gratuita y eliminar el problema de género en la educación. (PNUD, 2018c.)

En Guatemala la inaccesibilidad a la educación ha sido una forma de discriminación contra las mujeres y niñas, siendo la educación un derecho humano y básico. El empoderar a las mujeres y niñas tiene un efecto multiplicador y promotor del crecimiento económico y del desarrollo mundial (PNUD 2018d).

\section{Trabajo decente y reducción de desigualdades}

El octavo objetivo indica promover el crecimiento económico sostenido, inclusivo y sostenible, el trabajo decente para todos, es decir hombres y mujeres y la igualdad de remuneración, se debe de proteger los derechos laborales sobre todo el de las mujeres (Oficina internacional de trabajo OIT, 2015), en algunos lugares aún existen desigualdades en las oportunidades de trabajos donde a algunas se les niega esta oportunidad, y se les sigue explotando a través de la violencia y explotación sexual, siendo estos obstáculos difíciles de superar.

Al tener un mayor ingreso económico se mejora el poder adquisitivo en las familias logrando suplir más fácilmente las necesidades primarias como la alimentación, vivienda y vestuario.

Según la Encuesta Nacional de Empleo e Ingresos (2017) en Guatemala a nivel nacional se estima que el $70.2 \%$ por ciento de las personas trabajan en el sector informal lo que impide que tengan un salario fijo y digno y que puedan gozar de los beneficios de seguro social, vacaciones o bono entre otros, además muchas personas están subempleados no teniendo la oportunidad de suplir las necesidades básicas familiares y personales con lo cual se continua cerrando el círculo vicioso de pobreza, enfermedad, subdesarrollo.

\section{Resultados}

De los 17 ODS se puede identificar que: El fin de la pobreza, hambre cero, salud y bienestar, educación de calidad, igualdad de género, trabajo decente, crecimiento económico y reducción de las desigualdades, tienen influencia directa en disminuir la desnutrición en general y de las embarazadas.

Aunque Guatemala ha implementado diferentes políticas públicas en cada gobierno, no fueron alcanzados los objetivos del Milenio y los Objetivos de desarrollo sostenible siguen siendo un verdadero reto para alcanzar sin verse claramente si se está avanzando en ellos. 
La Pobreza en Guatemala para el 2016 era del 59.28 \% de la población de los cuales 23.33 \% en pobreza extrema, $35.93 \%$ en pobreza no extrema y un $40.72 \%$ en no pobreza, siendo estos mayores que los reportados en 2015 .

Según la Encuesta Nacional de Empleo e Ingresos (2017) en Guatemala a nivel nacional se estima que el $70.2 \%$ por ciento de las personas trabajan en el sector informal, lo que los priva de los beneficios que se tiene al estar en el sector formal como: seguro social, vacaciones, derecho a bonos etc, lo que repercute en la salud física y mental de las personas.

En Guatemala la inaccesibilidad a la educación ha sido una forma de discriminación contra las mujeres y niñas, siendo la educación un derecho humano y básico, aunque en los últimos años la brecha de educación entre hombres y mujeres ya se está cerrando lo cual puede ser un indicador de que las políticas de gobierno están alcanzando las metas propuestas.

\section{Conclusión}

La adecuada nutrición de las mujeres desde la infancia es importante ya que ésta repercutirá en sus descendientes, por lo cual se debe de trabajar arduamente en erradicar la pobreza para que toda la población tenga acceso a alimentación adecuada y eliminar la desigualdad de género para que las mujeres tengan las mismas oportunidades de alimentación, educación y trabajo que los hombres.

Es necesario que las mujeres embarazadas tengan acceso a la atención oportuna en sus controles prenatales para poder determinar con que índice de masa corporal están iniciando y logren alcanzar la ganancia de peso adecuada para la edad gestacional lo cual influirá en tener hijos con adecuado peso al nacer, disminuyendo la mortalidad neonatal.

La ventana de los 1000 días es una de las estrategias muy importantes del objetivo hambre cero, ya que la alimentación de las embarazadas y de sus hijos hasta los primeros dos años de vida influirá en el desarrollo de los niños tanto física como psicológicamente, dándole oportunidades de alcanzar todo su potencial.

La educación en mujeres es una prioridad, ya que está comprobado que las mujeres que tienen mayor grado de estudio se embarazan más tardíamente y tienen menor número de hijos, por lo que se debe de luchar por lograr el acceso de la educación para todos especialmente para las mujeres, dicha educación debe ser: inclusiva, equitativa y de calidad.

Alcanzar los Objetivos de desarrollo sostenible es una tarea difícil en la que se debe de comprometer cada gobierno, cada institución y cada individuo, hasta los últimos informes que se tienen en la VI ENSMI Guatemala está trabajando para lograr los objetivos de desarrollo sostenible habiendo mejoría pero aún no estamos cerca de lograrlo lo cual es una alerta de que si no se trabaja arduamente en ello no se volverán a alcanzar al igual que los objetivos del milenio. 
Es importante evaluar las políticas de gobierno y el cumplimiento de estas para evidenciar si son las más adecuadas o si se tienen que modificar para lograr los objetivos de desarrollo sostenible y cumplir los acuerdos hechos a nivel internacional.

Basado en los datos anteriores existe un alto riesgo de que al igual que no se cumplieron los objetivos del milenio no se logre alcanzar los objetivos de desarrollo sostenible y seguir con los mismos índices de desnutrición y muertes maternas y neonatales.

\section{Referencias}

Banco de Guatemala (2015). Guatemala en Cifras.

Banco de Guatemala (2017). Guatemala en Cifras.

Chan, M. (2010). La educación y la salud están íntimamente unidas. Organización Mundial de la Salud. Nueva York, Estados Unidos [Internet], 9, 20.

Constitución política de la república de Guatemala (1993). Decreto No. 18-93

Código de Salud de la república de Guatemala (1997). Decreto No. 90-97.

Comisión Nacional para la Reducción de la Desnutrición Crónica (2016). Estrategia para la prevención de la desnutrición crónica 2016-2020. Guatemala.

Delgado Álvarez, I., Roca Rosales, M. D. C., Suárez Vega, M., Rodríguez Alarcón, J. D., \& Ruiz Echavarría, Y. (2012). Repercusión de la desnutrición materna sobre el nacimiento de niños con bajo peso. Medisan, 16(10), 1478-1485.

Donoso, E., Carvajal, J. A., Vera, C., \& Poblete, J. A. (2014). La edad de la mujer como factor de riesgo de mortalidad materna, fetal, neonatal e infantil. Revista médica de Chile, 142(2), 168-174. https://doi. org/10.4067/S0034-98872014000200004

Fisher, M. (2015) La Malnutrición en Guatemala: Frenando el desarrollo de nuestro país.

Flórez, C. E., \& Soto, V. E. (2008). El estado de la salud sexual y reproductiva en América Latina y el Caribe: Una visión global. Inter-American Development Bank.

Fondo de las Naciones Unidas para la Infancia, (2010). Desnutrición, Recuperado de: https://www.unicef. org/guatemala/spanish/panorama_18467.htm

Gobierno de Guatemala. (2015). Informe ejecutivo Cuarto censo nacional de talla en escolares de educación primaria del sector público de la república de Guatemala.

Grados Valderrama, F. D. M., Cabrera Epiquen, R., \& Diaz Herrera, J. (2003). Estado nutricional pregestacional y ganancia de peso materno durante la gestación y su relación con el peso del recién nacido. Revista Medica Herediana, 14(3), 128-133. https://doi.org/10.20453/rmh.v14i3.2372

Instituto de Nutrición de Centro América y Panamá. (2017). El estado nutricional de las mujeres en Guatemala. Recuperado de : http://www.incap.int/index.php/es/noticias/1301-el-estado-nutricional-de-las-mujeres -en-guatemala

Instituto Nacional de Estadística (2017) Encuesta Nacional de Empleo e Ingresos - ENEI- 1-2017 
Instituto Nacional de Estadística (2017) VI encuesta Nacional de Salud Materno Infantil ENSMI 2014-2015.

Ley de Acceso Universal y Equitativo de Servicios de Planificación Familiar y su Integración en el Programa Nacional de Salud Reproductiva. (2005). Decreto Número 87-2005.

Ley del Sistema Nacional de Seguridad Alimentaria y Nutricional (2005). Decreto número 32-2005.

Ley de Protección Integral de la Niñez y la Adolescencia, Decreto número 27-2003.

Ley de Protección para las Personas de la Tercera Edad (1996). Decreto número 80-1996.

Mendoza, L., Pérez, B., \& Sánchez Bernal, S. (2010). Estado nutricional de embarazadas en el último mes de gestación y su asociación con las medidas antropométricas de sus recién nacidos. Pediatría (Asunción), 37(2), 91-96.

Ministerio de Salud Pública y Asistencia Social. (2010). Vigilancia de la Embarazada y de la Muerte de Mujeres en Edad Fértil (10 a 54 años) para la identificación de las muertes maternas. Guatemala.

Morlachetti, A. (2007). Políticas de salud sexual y reproductiva dirigidas a adolescentes y jóvenes: un enfoque fundado en los derechos humanos. Notas de población.

Naciones Unidas. (2016). Agenda 2030 y los Objetivos de Desarrollo Sostenible Una oportunidad para América Latina y el Caribe.

Naciones Unidas. (2017). Informe de los Objetivos de Desarrollo Sostenible

Oficina Internacional de trabajo. (2015). La Agenda 2030 para el Desarrollo Sostenible. Consejo de Administración 325. reunión, Ginebra, 29 de octubre - 12 de noviembre

Organización Panamericana de la Salud (2009). Desnutrición en Guatemala 2009. Situación actual: perspectivas para el fortalecimiento del sistema de vigilancia nutricional.

Organización de las Naciones Unidas para la alimentación y loa agricultura. (2015). La FAO y los 17 Objetivos de Desarrollo Sostenible.

Organización de la Naciones Unidas Mujeres (sf). Las mujeres y los Objetivos de Desarrollo Sostenible. Recuperado de: http://www.unwomen.org/es/news/in-focus/women-and-the-sdgs

Organización de las naciones unidas, Mujeres. (2017) ODS 5: Lograr la igualdad entre los géneros y empoderar a todas las mujeres y las niñas http:/www.unwomen.org/es/news/in-focus/ women-and-the-sdgs/sdg-5-gender-equality

Peraza Roque, G. J., Pérez Delgado, S. D. L. C., \& Figueroa Barreto, Z. D. L. A. (2001). Factores asociados al bajo peso al nacer. Revista Cubana de Medicina General Integral, 17(5), 490-496.

Pimiento Infante, L. M., \& Beltrán Avendaño, M. A. (2015). Restricción del crecimiento intrauterino: una aproximación al diagnóstico, seguimiento y manejo. Revista chilena de obstetricia y ginecología, 80(6), 493-502. https://doi.org/10.4067/S0717-75262015000600010

Programa de las Naciones Unidas para el Desarrollo Guatemala. (2015). Objetivos de Desarrollo Sostenible. Recupero de: http://www.gt.undp.org/content/guatemala/es/home/sustainable-development-goals.html 
Programa de las Naciones Unidas para el Desarrollo (2018a). Objetivos de Desarrollo Sostenible. Recuperado de: http://www.gt.undp.org/content/guatemala/es/home/sustainable-development-goals. html

Programa de las Naciones Unidas para el Desarrollo, Guatemala. (2018b ). Hambre cero. Recuperado de: http://www.gt.undp.org/content/guatemala/es/home/sustainable-development-goals/goal-2-zero-hunger.html

Programa de las Naciones Unidas para el Desarrollo Guatemala (2018c) Educación de calidad. Recuperado de : http://www.gt.undp.org/content/guatemala/es/home/sustainable-development-goals/ goal-4-quality-education.html

Programa de las Naciones Unidas para el Desarrollo, Guatemala (2018d). Igualdad de Género. Recuperado de: http://www.gt.undp.org/content/guatemala/es/home/sustainabledevelopment-goals/goal-5gender-equality.html

Rebollar, E., Ávila, J., \& Gutiérrez, V. (2010). Factores de riesgo asociados al bajo peso al nacer. Investig Materno Infant, 2(3), 117-22.

Rivera, J. A., Martorell, R., González, W., Lutter, C., Cossío, T. G. D., Flores-Ayala, R., ... \& Delgado, H. (2011). Prevención de la desnutrición de la madre y el niño: el componente de nutrición de la Iniciativa Salud Mesoamérica 2015. salud pública de méxico, 53, 303-311.

Sánchez Salazar, F. R., Trelles Aguabella, E., Castanedo Valdés, R., \& Lugones Botell, M. (2003). Índice de masa corporal en embarazadas adolescentes. Revista Cubana de Medicina General Integral, 19(1), 0-0.

Secretaría de Planificación y Programación de la Presidencia, SEGEPLAN (2015). Informe final de cumplimiento de objetivos del milenio.

Secretaría de Planificación y Programación de la Presidencia, SEGEPLAN (2016). Infografía de los Objetivos del Milenio a los Objetivos de desarrollo sostenible.

Secretaría de Planificación y Programación de la Presidencia, SEGEPLAN (2016). Estrategia de articulación de la Agenda de Objetivos de Desarrollo Sostenible con el Plan y la Política Nacional de Desarrollo K'atun Nuestra Guatemala 2032

Secretaría de Planificación y Programación de la Presidencia, SEGEPLAN (2017). Objetivos de desarrollo sostenible, Metas priorizadas.

Scalin Up Nutrition. (2015) La nutrición y los Objetivos de desarrollo sostenible. Recuperado de http:// scalingupnutrition.org/es/nutricion/la-nutricion-y-los-objetivos-de-desarrollo-sostenible/

Vivero,J. (2014) Informe de Consultoría Apoyo a la sostenibilidad de los ejes principales de acción del Plan del Pacto Hambre Cero en el marco de la política de seguridad alimentaria y nutricional del país.

Wisbaum, W. (2011). La desnutrición infantil: causas, consecuencias y estrategias para su prevención y tratamiento. 


\section{Sobre la autora}

\section{Yessenia Eunice Recinos Méndez}

Médico y Cirujano con especialidad en Ginecología y Obstetricia egresada de la Facultad de Ciencias Médicas, Universidad de San Carlos de Guatemala Estudia Doctorado en investigación para el desarrollo social en el Centro Universitario de Suroriente de Jalapa.

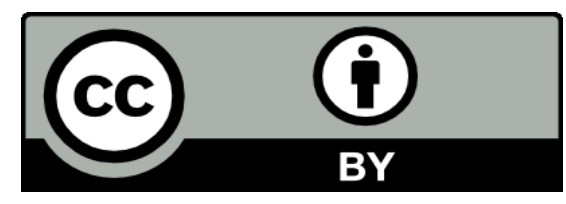

Este texto está protegido por una licencia CreativeCommons 4.0.

Usted es libre para compartir, copiar y redistribuir el material en cualquier medio o formato y adaptar el documento, remezclar, transformar y crear a partir del material para cualquier propósito, incluso comercialmente, siempre que cumpla la condición de atribución: usted debe reconocer el crédito de una obra de manera adecuada, proporcionar un enlace a la licencia, e indicar si se han realizado cambios. Puede hacerlo en cualquier forma razonable, pero no de forma tal que sugiera que tiene el apoyo del licenciante o lo recibe por el uso que hace. 